M. Stöhr, C. M. Arndt and W. Meier, Transient effects of fuel-air mixing in a partially-premixed turbulent swirl flame, Proceedings of the Combustion Institute 35 (2015) 3327-3335.

The original publication is available at www.elsevier.com

http://dx.doi.org/10.1016/j.proci.2014.06.095 


\title{
Transient effects of fuel-air mixing in a partially-premixed turbulent swirl flame
}

\author{
M. Stöhr ${ }^{\mathrm{a}, *}$, C. M. Arndt ${ }^{\mathrm{a}}$, W. Meier ${ }^{\mathrm{a}}$ \\ ${ }^{a}$ German Aerospace Center (DLR), Institute of Combustion Technology, Pfaffenwaldring 38-40, 70569 Stuttgart, Germany
}

\begin{abstract}
Unsteady interactions of flow, fuel-air mixing and reaction in a lean partially-premixed turbulent swirl flame are investigated using simultaneous particle image velocimetry and planar laser-induced fluorescence of $\mathrm{OH}$ and acetone with a repetition rate of $10 \mathrm{kHz}$. The flame is operated with methane and air in a gas turbine model combustor at ambient temperature and pressure. Transport and mixing of fuel is visualized by fluorescence of acetone, which is added with $9 \%$ by volume to the methane. The dominant unsteady flow structures are a precessing vortex core (PVC) in the shear layer of the inner recirculation zone and the lower stagnation point (LSP) at the flame root. The measurements show that fuel and air are largely separated at the inlet of the combustion chamber, but are then strongly mixed by the PVC. A well-mixed zone of unburned fuel and air is formed around the vortex, and then ignited by recirculating burned gas. At the flame root, the PVC induces periodic changes in the composition of the unburned gas, which varies between pure air and well-mixed fuel and air. Reaction is locally quenched at the LSP when fresh air without fuel is present, and re-ignition takes place when mixed fuel and air arrive at the boundary of recirculating burned gas. Generally, the results show that the enhancement of fuel-air mixing induced by the PVC contributes significantly to the stabilization of the flame, and that the flame dynamics can only be properly understood when an analysis of transient mixing mechanisms is included.
\end{abstract}

Keywords: Turbulent swirl flames, Fuel-air mixing, Precessing vortex core, Gas turbine combustion, High-speed laser diagnostics

\section{Introduction}

In gas turbine (GT) combustors, the mode of fuel injection and premixing largely influences the level of $\mathrm{NO}_{x}$ emissions $[1,2,3]$ and the occurence of thermoacoustic oscillations [2, 4], blowout [2] or flashback. While lean flames with perfect premixing generally provide the lowest $\mathrm{NO}_{x}$ emissions, they are rather susceptible to thermoacoustic oscillations or flashback. The combustors are therefore often operated in a partially premixed or piloted premixed mode, where the unburned gas exhibits spatio-temporal variations of equivalence ratio, and fuelair mixing continues within the flame zone.

In turbulent swirl flames, which are commonly used in GT burners, mixing results not only from turbulent fluctuations, but is often increased by coherent flow structures such as the so-called precessing vortex core (PVC) [5]. For non-reacting conditions, enhanced fuel-air mixing due to the PVC has been demonstrated using DNS [6] and LES [7]. Under reacting conditions, the situation is much more complex because the PVC not only causes mixing of fuel and air [8], but also mixing of burned and unburned gas [9]. In addition, the PVC has direct effects on the flame such as flame roll-up, flame stretch or local quenching $[10,11]$. The direct effects, in turn, depend on local equivalence ratio and temperature, and thus on the dynamics of mixing.

Generally, these transient interactions of flow, mixing and reaction in turbulent swirl flames are still not well understood, and

${ }^{*}$ Corresponding author. Email: michael.stoehr@dlr.de further improvements of GT combustors with respect to emissions or reliability therefore require additional studies. Previous experimental studies of fuel-air mixing in turbulent swirl flames, however, were limited to pointwise measurements or low repetition rates that cannot resolve the temporal dynamics $[8,12,13,14,15]$.

The present work investigates the dynamics and effects of fuel-air mixing in a turbulent swirl flame operated in a GT model combustor. Using simultaneous particle image velocimetry (PIV) and planar laser-induced fluorescence (PLIF) of acetone and $\mathrm{OH}$ with a repetition rate of $10 \mathrm{kHz}$, transient interactions of flow, mixing and reaction are measured in a 2D domain. The work continues a series of previous studies in the same combustor aiming at a detailed understanding of the stabilization mechanisms of confined turbulent swirl flames $[14,15,16]$. The existence and the properties of the PVC in this combustor have been determined by experiments $[9,10]$ and numerical simulation [17]. Most recently, simultaneous timeresolved PIV and OH-PLIF with up to $10 \mathrm{kHz}$ has been applied for studies of PVC-flame interaction [10,11]. OH-PLIF, however, only provides information about regions where reaction has taken place. The system is therefore now extended by tracer-PLIF for visualization of fuel transport and mixing in the unburned gas. As a fluorescent tracer, $9 \%$ acetone by volume is added to the methane that is used as fuel. The analysis focuses on the regions that are most critical for the stabilization mechanism, namely the inner shear layer and the flame root. 


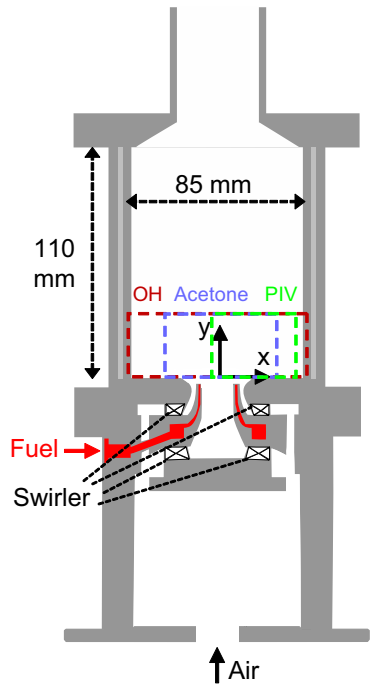

a Side view of combustor

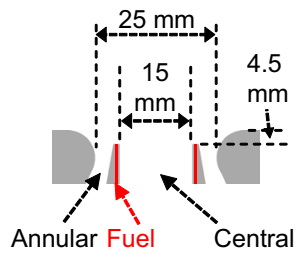

nozzle channels nozzle

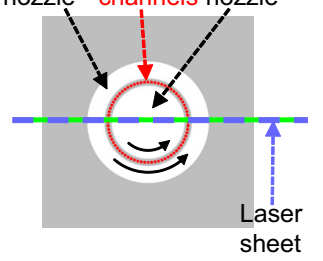

b Side and top view of air nozzles and fuel injection
Figure 1: Geometry of gas turbine model combustor.

\section{Experimental setup}

\subsection{Combustor and operating condition}

The geometric design of the gas turbine model combustor is shown in Fig. 1. Air first enters a plenum (diameter $d=79 \mathrm{~mm}$, height $h=65 \mathrm{~mm}$ ) and then separately passes two radial swirl generators. The two co-swirling flows enter the combustion chamber through a central nozzle $(d=15 \mathrm{~mm})$ and an annular nozzle $(d=25 \mathrm{~mm})$. Gaseous fuel is fed through 72 channels $\left(0.5 \times 0.5 \mathrm{~mm}^{2}\right)$ forming a ring between the air nozzles. The exit planes of the fuel and central air nozzles are located $4.5 \mathrm{~mm}$ below the exit plane of the outer air nozzle which is defined as $y=0 \mathrm{~mm}$. The combustion chamber has a square cross-section of $85 \times 85 \mathrm{~mm}^{2}$, a height of $114 \mathrm{~mm}$, and is enclosed by four quartz windows allowing optical access for lasers and cameras. A conical top plate with a central exhaust tube $(d=40 \mathrm{~mm}, h=50$ $\mathrm{mm}$ ) forms the exit.

The burner was operated with methane/acetone and dry air at ambient temperature and pressure. The methane was divided into a bypass and a seeding flow. The seeding flow was bubbled through a series of two temperature-controlled gaswashing bottles $\left(T=25^{\circ} \mathrm{C}\right)$ filled with acetone, so that a saturated methane/acetone mixture was achieved. The two flows were again combined and directed to the burner through a heated pipe $\left(T=35^{\circ} \mathrm{C}\right)$ to avoid condensation. The acetone fraction in the fuel was controlled by varying the bypass/seeding ratio. For the measurements, an acetone volume fraction of $9 \%$ was chosen. This provided a good PLIF signal intensity while the influence of acetone on the flame is low as shown later in Sect. 3.3.

Several non-reacting and reacting conditions have been measured with the present setup. This study, however, focuses on one single reacting case with a global equivalence ratio of $\phi=0.65$ and a thermal power of $P=10 \mathrm{~kW}$ (based on the mixture of methane, acetone and air). The average velocity of air in the nozzle is $8.9 \mathrm{~m} / \mathrm{s}$, and the velocity of the fuel at the exit of the fuel channels is $15.3 \mathrm{~m} / \mathrm{s}$. The Reynolds number of the unburned inflow is $\mathrm{Re}=15000$ and the swirl number was estimated in a previous work as $S \approx 0.55$ [14].

\subsection{Measuring techniques}

The measuring setup is based on a previously used PIV and OH-PLIF system with a recording rate of $f_{\text {rec }}=10 \mathrm{kHz}$, which was now extended by acetone-PLIF. Below the PIV and OHPLIF system is briefly described (further details can be found in Refs. $[16,11])$, and the extensions regarding acetone-PLIF are specified. Issues regarding the analysis and interpretation of acetone-PLIF data are discussed in Sect. 3.

The PIV system consisted of a dual-cavity Nd:YAG laser (Edgewave IS-6IIDE, $2.6 \mathrm{~mJ} /$ pulse at $532 \mathrm{~nm}$ ) and a CMOS camera (LaVision HSS 6). OH-PLIF and acetone-PLIF were excited by the same dye laser (Sirah Credo, $100 \mu \mathrm{J} /$ pulse at $283.2 \mathrm{~nm}$ ) pumped with a Nd:YAG laser (Edgewave IS-8IIE, 4 $\mathrm{mJ} /$ pulse at $532 \mathrm{~nm})$. The dye laser was tuned to the $\mathrm{Q}_{1}(7)$ line of the A-X $(1,0)$ transition of $\mathrm{OH}$ near $283.2 \mathrm{~nm}$. The beams of the two laser systems were expanded into two coplanar vertical light sheets across the central section $(z=0 \mathrm{~mm})$ of the combustion chamber (Fig. 1b). In the chamber, the thickness of the laser sheets was $0.7 \mathrm{~mm}$ and $0.4 \mathrm{~mm}$ for PIV and PLIF, respectively.

The resulting emission spectra of OH-PLIF (300-325 nm) and acetone PLIF (350-550 $\mathrm{nm}$ [21]) are well separated. The OH-PLIF was recorded using an intensified CMOS camera (LaVision HSS 5 with HS-IRO) equipped with a UV lens (Cerco, $f=45 \mathrm{~mm}, \mathrm{f} / 1.8)$ and a bandpass filter (300-325 nm). The acetone-PLIF was recorded using an intensified CMOS camera (LaVision HSS 8 with HS-IRO) equipped with a camera lens (Canon, $f=85 \mathrm{~mm}, \mathrm{f} / 1.2$ ). Below $350 \mathrm{~nm}$, the transmission of the lens is virtually zero, and therefore no additional filter was needed to block the OH-PLIF emission. Image sequences of $\mathrm{OH}$ chemiluminescence $(\mathrm{CL})$ were recorded using the OH-PLIF detection system with an exposure time of $20 \mu \mathrm{s}$.

The field of view for the PIV was limited to a $40 \times 30 \mathrm{~mm}^{2}$ region above the nozzle (Fig. 1a). The PLIF fields also extended up to a height of $y=30 \mathrm{~mm}$, but across the full width for $\mathrm{OH}$, and within $-25 \mathrm{~mm}<x<28 \mathrm{~mm}$ for acetone. All cameras had sufficient on-board memory for 7500 individual recordings corresponding to an acquisition period of $0.75 \mathrm{~s}$. The PIV images were processed using a cross-correlation algorithm (LaVision $\mathrm{DaVis}$ 8.1) with a final interrogation window size of 16 $\times 16$ pixel, resulting in velocity fields with an in-plane spatial resolution of $1.2 \times 1.2 \mathrm{~mm}^{2}$. The average PLIF laser sheet profile inhomogeneities were corrected using a sheet profile that was recorded separately when the chamber was homogeneously filled with acetone vapor.

For the present operating condition, the chemical time scale $\tau_{\mathrm{c}}$ and the flow time scale regarding the PVC $\tau_{\mathrm{f}}$ have been estimated in Ref. [11] as $\tau_{\mathrm{c}}=2 \mathrm{~ms}$ and $\tau_{\mathrm{f}}=2 \mathrm{~ms}$ (i.e., Damköhler number $\mathrm{Da}=\tau_{\mathrm{f}} / \tau_{\mathrm{c}}=1$ ). These time scales are well resolved by the measurements with time steps of $\mathrm{dt}=1 / f_{\text {rec }}=0.1 \mathrm{~ms}$. 


\subsection{Measurement uncertainties}

In the following, the most relevant measuring uncertainties for PIV and acetone-PLIF are discussed. Since OH-PLIF is used only as a qualitative marker of flame structure, we omit a discussion of uncertainties for this technique.

Because only one camera was available for PIV, only inplane velocities were measured. In this case, the perspective error $\Delta v_{\text {persp }}$ (which does not occur for stereo-PIV) has to be considered. The value of $\Delta v_{\text {persp }}$ depends on the (unknown) outof-plane motion $v_{z}$ and the angle of view $\varphi$ as $\Delta v_{\text {persp }}=v_{z} \tan \varphi$ [22]. For the present configuration, $\tan \varphi$ is $\approx 0.5$. Previous stereo-PIV measurements [10] showed that for the present flame, local values of $\left|v_{z}\right|$ can be up to $10 \mathrm{~m} / \mathrm{s}$ in the swirling jet near the nozzle, whereas for other regions $\left|v_{z}\right|$ is below $5 \mathrm{~m} / \mathrm{s}$. Local values of $\Delta v_{\text {persp }}$ are thus mainly below $2.5 \mathrm{~m} / \mathrm{s}$, but can be up to $5 \mathrm{~m} / \mathrm{s}$ in the jet. A second uncertainty of velocities stems from the \pm 0.1 pixel uncertainty of the PIV peak-finding algorithm. The resulting random uncertainty of in-plane instantaneous velocities is estimated as $\pm 0.6 \mathrm{~m} / \mathrm{s}$.

Regarding acetone-PLIF, uncertainty results from shot-toshot fluctuations of laser pulse energy, which cause a corresponding variability of total LIF image intensity, and were determined as $6.5 \%$. A second source of uncertainty is the $\mathrm{CH}-\mathrm{CL}$ that occurs in the region of heat release (cf. Fig. 4). $\mathrm{CH}$ emits in the range $420-440 \mathrm{~nm}$ and overlaps with the acetone-PLIF emission (350-550 nm). The intensifier gate time (100 ns) was chosen as short as possible to reduce the signal of $\mathrm{CH}$. The remaining $\mathrm{CH}$ signal was on the order of 50 counts, and thus low compared to the acetone signals of up to 2000 counts depending on the local equivalence ratio. Further issues concerning the interpretation of acetone-PLIF images are addressed in Sect. 3.

For the purpose of validation of numerical simulations, the present uncertainties are quite large. The present work, however, mainly examines the interaction of flow structures, mixing patterns and flame dynamics in a qualitative way. For this purpose, the uncertainties are acceptable. Accurate data for model validation in this combustor has been provided in several previous works $[14,15,10]$.

\section{Characteristics of acetone-PLIF}

\subsection{Temperature dependence and pyrolysis}

In the present combustor, the temperature of unburned fuelair mixtures may increase by several $100 \mathrm{~K}$ due to heat transfer from recirculating burned gas $[14,15]$, and therefore it is necessary to consider the effect of temperature on acetone fluorescence. For excitation at $283.2 \mathrm{~nm}$, the fluorescence per unit mole fraction strongly decreases with temperature according to the results by Thurber et al. [23] shown in Fig. 2a. Since the temperature is not measured, the mole fraction and thus the local equivalence ratio cannot be estimated from the acetone PLIF signal. Therefore, the study of fuel-air mixing in Sect. 5 is limited to a qualitative analysis.

Elevated temperature may also cause pyrolysis of acetone. Time constants of acetone pyrolysis at atmospheric pressure reported by Yip et al. [24] are plotted in Fig. 2b. The time constant increases exponentially with decreasing temperature and
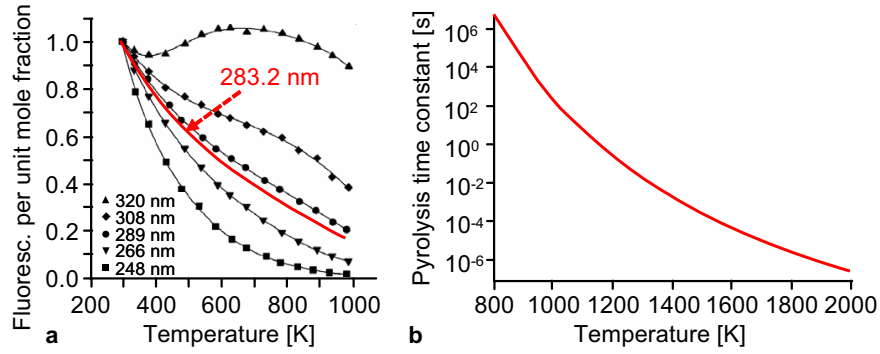

Figure 2: Temperature dependence of (a) acetone fluorescence (adopted from Ref. [23]) and (b) acetone pyrolysis (data from Ref. [24]).

is $>100 \mathrm{~s}$ for all $T<1000 \mathrm{~K}$. Raman spectroscopy measurements in the present combustor $[14,15]$ have shown that the temperature of unburned gas remains typically well below $1000 \mathrm{~K}$, and therefore effects of pyrolysis can be neglected.

\subsection{Differential diffusion}

Since the molecular diffusivity in air of methane $\left(\alpha_{\mathrm{Me}}=0.3\right.$ $\left.\mathrm{cm}^{2} / \mathrm{s}\right)$ differs from that of acetone $\left(\alpha_{\mathrm{Ac}}=0.1 \mathrm{~cm}^{2} / \mathrm{s}\right)$, they generally mix differentially into the ambient air. The fluorescence of acetone may thus not accurately represent the distribution of methane, and it is therefore desirable to estimate the magnitude of their differential mixing. In a recent experimental study, Brownell \& Su [25] investigated differential diffusion from a jet of acetone and helium $\left(\alpha_{\mathrm{He}}=0.8 \mathrm{~cm}^{2} / \mathrm{s}\right)$ in an air coflow. They found that at length scales larger than the jet nozzle diameter, the relative differences between acetone and helium concentrations are typically less than $5 \%$, while at smaller scales differences up to $10 \%$ may occur. In the present study, the ratio of diffusivities, $\alpha_{\mathrm{Ac}} / \alpha_{\mathrm{Me}}=3$, is considerably smaller than in the study of Ref. [25], $\alpha_{\mathrm{Ac}} / \alpha_{\mathrm{He}}=8$. This suggests that for length scales larger than the fuel channel width $(d=0.5 \mathrm{~mm})$, differences of acetone and methane concentration are significantly lower than $5 \%$ in the present experiment.

\subsection{Effects and behavior of acetone in methane-air flames}

It is important that the added acetone does not manipulate the flame, so that conclusions on the behavior of the original flame without acetone can be drawn. In the following, different effects regarding the behavior of acetone in the flame are discussed.

One important value is the laminar flame speed $S_{\mathrm{L}}$. Chong \& Hochgreb [26] have measured $S_{\mathrm{L}}$ for methane-acetone-air mixtures with different levels of acetone in the fuel, including $9 \%$ by volume like in the present work. In the range $0.6<\phi<1.4$, $S_{\mathrm{L}}$ varies between 15 and $40 \mathrm{~cm} / \mathrm{s}$. The differences of $S_{\mathrm{L}}$ for $0 \%$ and $9 \%$ acetone are within the measurement uncertainty of $1.5 \mathrm{~cm} / \mathrm{s}$ for $\phi<1.25$, and below $5 \mathrm{~cm} / \mathrm{s}$ for $\phi>1.25$. Since the present flame is operated at $\phi_{\text {global }}=0.65$, no major changes of $S_{\mathrm{L}}$ are expected for the $9 \%$ acetone level used in this work.

Model calculations by Chong \& Hochgreb also showed that for an acetone level of $10 \%$, the spatial profiles of methane and acetone coincide within $0.1 \mathrm{~mm}$ in the reaction zone and that no significant changes for temperature and heat release profiles occur. This indicates that the addition of $\leq 10 \%$ acetone 


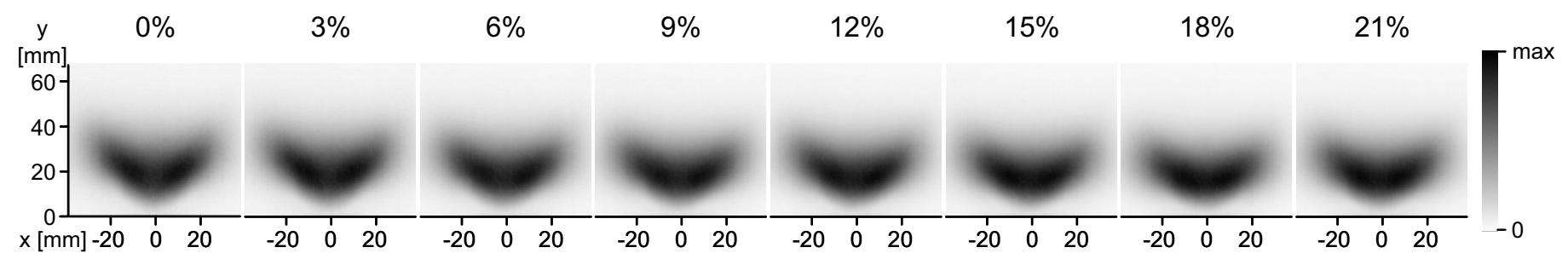

Figure 3: Average flame shape for different acetone contents in the fuel.

does not significantly change the reaction of methane and air. It also shows that acetone not only represents the transport of methane in the unburned gas, but is also a good reactive marker for methane, and thus expected to be present only in the unburned gas.

In order to examine the effect of acetone on the present turbulent swirl flame, OH-CL images have been recorded for acetone contents between $0 \%$ and $21 \%$. The averaged OH-CL images, which are considered as a line-of-sight integrated marker of heat release [19], are shown in Fig. 3. As expected from the findings discussed above, the effect of acetone on the flame shape is quite small. As the acetone content increases from $0 \%$ to $21 \%$, the flame height decreases slightly by $\approx 2 \mathrm{~mm}$, which might be related to the corresponding slight increase of laminar flame speed [26]. As a dynamic flame feature, the frequency of the PVC (described in Sect. 4.3) remains contant within \pm 2 Hz. Altogether, the above results indicate that the addition of $9 \%$ aceton by mole has no major effect on the flame, and the acetone-seeded flame is thus a good representative of the unseeded methane-air flame.

\section{Flow field and flame shape}

\subsection{Flame shape}

Figure 4a shows an average OH-CL image of the flame with $9 \%$ acetone. Due to the rotational symmetry of the flame, the original line-of-sight-integrated image has been deconvolved by an Abel inversion to derive the distribution in the plane through the center of the flame. It is seen that the flame is lifted $\approx 10 \mathrm{~mm}$ above the exit of the fuel channels $(y=-4.5 \mathrm{~mm})$. Over this distance, fuel and air start mixing, and the flame is therefore considered as partially premixed. Heat release takes place in a conically shaped zone within a radius of $r<35 \mathrm{~mm}$ and up to a height of $y=40 \mathrm{~mm}$.

\subsection{Average flow field}

For the characterization of the flow field, velocity measurements over the full cross-section of the chamber are presented below. These have been obtained in a previous study [10] using stereo-PIV for the flame without acetone (no major changes of flow field compared to the case with 9\% acetone are expected according to the discussion in Sect. 3.3).

The structure of the average flow field shown in Fig. $4 \mathrm{~b}$ is typical of confined swirl flames. From the nozzle at the bottom, unburned fuel and air enter the chamber in the form of a coneshaped stream. Part of the burned gas flows back towards the nozzle through the inner (IRZ) and outer (ORZ) recirculation zones. This provides a source of heat and radicals to the unburned gas, which enhances ignition and thus facilitates flame stabilization. Strong velocity gradients occur in the inner shear layer (ISL) between the inflow and the IRZ, and in the outer shear layer (OSL) between inflow and ORZ. The average lower stagnation point (LSP), i.e. the upstream boundary of the IRZ, is located below the measurement domain $(y<0 \mathrm{~mm})$.

\subsection{Precessing vortex core}

Figure $4 \mathrm{c}$ shows a typical instantaneous velocity field. The most distinctive feature compared to the average flow is the zigzag arrangement of vortices in the ISL marked by the dashed line. This arrangement indicates the presence of a coherent 3D helical vortex in the ISL. Such a helical vortex is found in many GT-typical swirl flames (literature surveys are provided in Refs. $[5,10])$ and is commonly termed a precessing vortex core (PVC). It rotates around the central axis $(x=z=0 \mathrm{~mm})$ with a frequency of about $500 \mathrm{~Hz}$ for the present condition. The upper part of the PVC is located in the flame zone, and affects the flame through flame roll-up and local increase of heat release as shown in previous studies $[9,10,11]$. The lower part extends into the region of unburned gas $(y<5 \mathrm{~mm})$, and thus also affects mixing of fuel and air as it will be seen in Sect. 5.2.

\subsection{Unsteady lower stagnation point}

Another important feature is the unsteady LSP (marked with a black dot), which differs from the LSP of the average flow field. The former lies at the center of the flame root, where hot burned gas and fresh gas collide frontally (marked with curved arrows). In this zone, heat and radicals are formed, which contribute to the stabilization of the flame in the ISL as shown in Ref. [10]. On the other hand, the opposed flow causes high strain rates, which can cause transient local extinctions as shown in Refs. [16, 18]. The opposed-flow zone is coupled to the PVC and thus also rotates around the cental axis [10]. It will be seen in Sect. 5.3 that the flame near the unsteady LSP is subject to strong fluctuations of local equivalence ratio. 

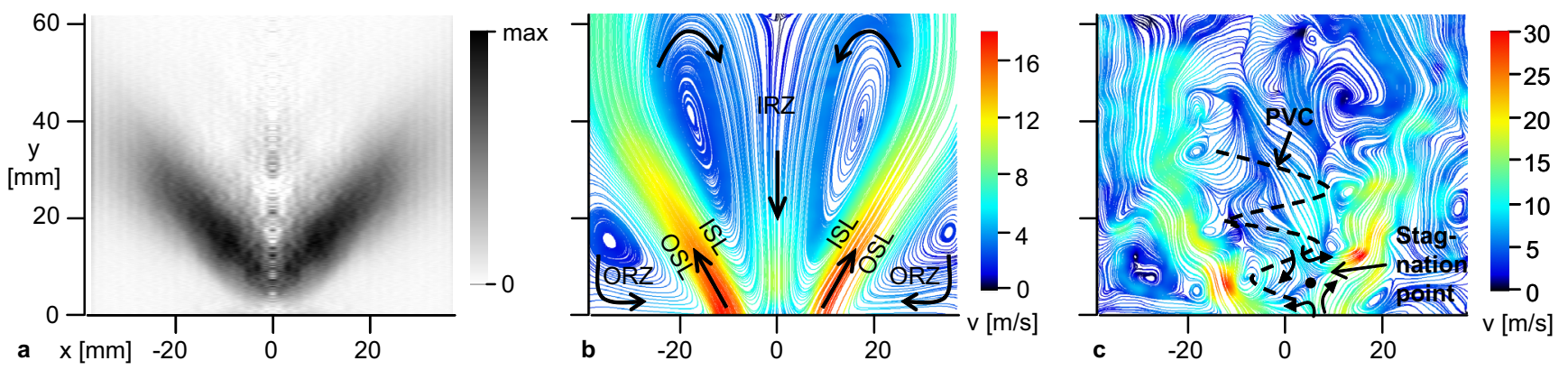

Figure 4: (a) Average flame shape (abel-deconvoluted), (b) average flow field, and (c) instantaneous flow field.

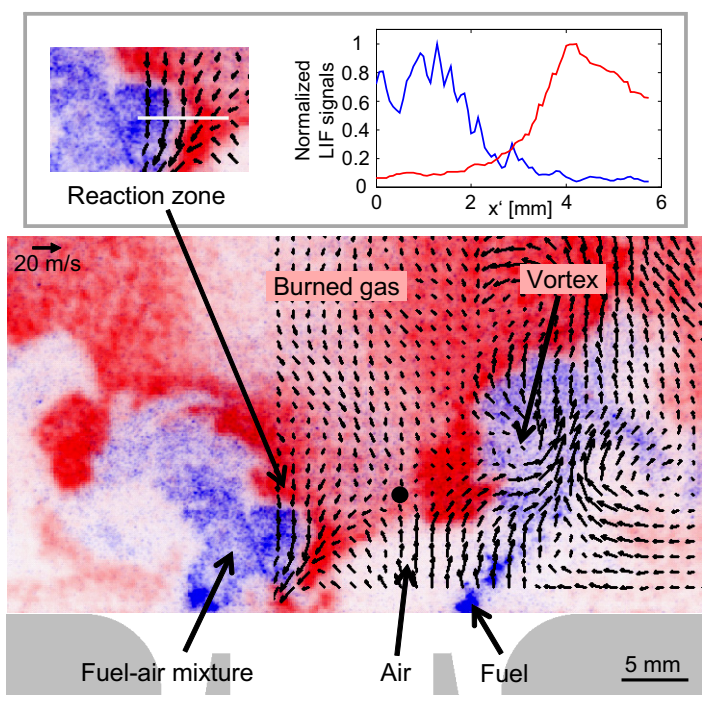

Figure 5: Simultaneous PIV, OH-PLIF (red) and acetone-PLIF (blue) measurement.

\section{Dynamics and interplay of fuel-air mixing and reaction}

\subsection{Instantaneous flame structure, fuel distribution and flow field}

Figure 5 shows a typical simultaneous measurement of velocity field, OH-PLIF and acetone-PLIF. The velocity field exhibits the typical features associated with a PVC, i.e., the vortex in the ISL (marked with an arrow) and the unsteady LSP (marked with a dot) surrounded by an opposed-flow zone.

In the distribution of $\mathrm{OH}$ shown in red, different zones within the flame can be distinguished depending on the level of $\mathrm{OH}$. Regions without $\mathrm{OH}$ (white or blue) represent gas with low or medium temperature $(T<1500 \mathrm{~K})$, i.e., unburned gas with possible admixture of burned gas. High levels of $\mathrm{OH}$ (dark red) indicate superequilibrium $\mathrm{OH}$, which is formed in the reaction zones and has a half-life period of $\approx 1 \mathrm{~ms}$ under atmospheric pressure [20]. Regions with medium and low levels of $\mathrm{OH}$ (medium and light red) represent burned gas whose $\mathrm{OH}$ concentration has decayed toward equilibrium while it was transported away from the reaction zone. The equilibrium $\mathrm{OH}$ concentration increases with temperature and as the stoichiometric condition is approached [20].
The acetone-PLIF signal represented in blue shows the distribution of fuel in the unburned gas. Strong variations in the degree of mixing of fuel and air are visible. Near the nozzle on the bottom right, a region of pure air (white) and a streak of fuel-rich gas (dark blue) are seen. Around the vortex, on the other hand, a zone of well-mixed fuel and air (medium and light blue) has formed.

As pointed out in Sect. 3.3, acetone and methane react almost simultaneously under the present conditions, and thus no acetone is expected in the burned gas. A region where reaction takes place is highlighted in upper part of Fig. 5. The (normalized) profiles along the white line show the typical behavior of fuel and $\mathrm{OH}$ across a reaction zone: $\mathrm{As} \mathrm{OH}$ is produced during reaction, the level of $\mathrm{OH}$ starts rising at $x^{\prime}=2 \mathrm{~mm}$ until it reaches a maximum at $x^{\prime}=4 \mathrm{~mm}$. In the burned gas $\left(x^{\prime}>4 \mathrm{~mm}\right)$, the superequilibrium $\mathrm{OH}$ decays to equilibrium. As expected, the acetone level falls off in the same zone where $\mathrm{OH}$ increases. As noted in Sect. 2.3, low levels of acetone-PLIF that are seen in some places of burned gas are caused by $\mathrm{CH}-\mathrm{CL}$ emissions.

In the following, the interaction of flow, mixing and reaction is studied using time-resolved measurements. The analysis focuses on the two regions that are critical for the stabilization of the flame, i.e., the ISL (Sect. 5.2) and the flame root (Sect. 5.3).

\subsection{Vortex-induced mixing and reaction in the ISL}

Figure 6 shows an exemplary time-series of simultaneous measurements of velocity field, OH-PLIF and acetone-PLIF. The following analysis focuses on the area around the ISL on the right side where the velocity field is measured.

At $t=0 \mathrm{~ms}$, separated streams of air and fuel (marked by arrows) enter the chamber from the nozzle at the bottom. At $t=0.4$ $\mathrm{ms}$, a vortex appears at the bottom and entrains the fuel towards the center $(x=0 \mathrm{~mm})$. The vortex is part of the helical PVC, and moves downstream in the following images due to the rotation of the PVC helix around the burner axis. The vortex causes strong mixing of fuel and air at $t=0.8 \mathrm{~ms}$, whereas unmixed streams of air and fuel are now seen at the bottom left. At $t=1.3$ $\mathrm{ms}$ and $t=1.9 \mathrm{~ms}$, the vortex moves further downstream and induces roll-up of the flame. During roll-up, the level of $\mathrm{OH}$ increases, which indicates an enhanced rate of heat release. At the same time, the amount of fuel around the vortex declines. At $t=2.2 \mathrm{~ms}$, heat release takes place in a large area around the 


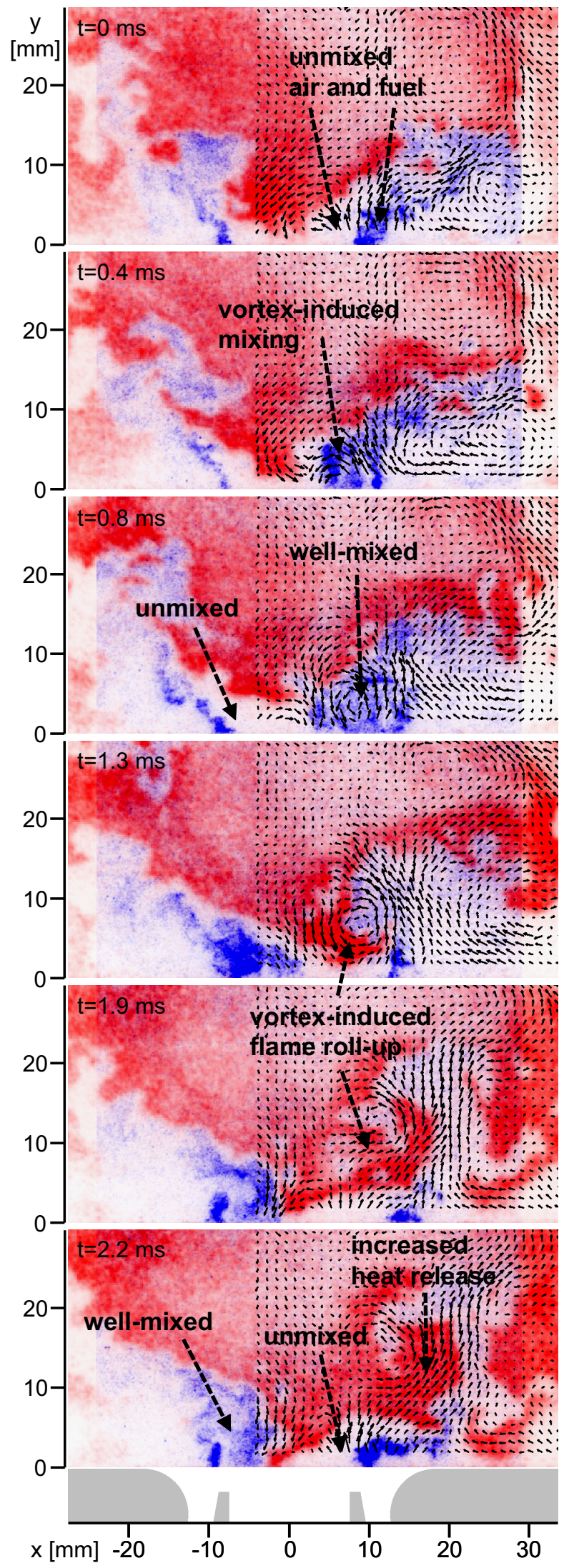

Figure 6: Time-series of PIV, OH-PLIF and acetone-PLIF measurements showing vortex-induced mixing and reaction in the ISL. For clarity reasons, not all time steps are shown. vortex, while the fuel has been largely consumed. In the following frames (not shown), similar vortex-induced mixing and heat release recurs periodically with the $\mathrm{PVC}$ frequency of $\approx 500 \mathrm{~Hz}$ on the left and right side of the ISL.

The vortex-induced flame roll-up starting at $t=1.3 \mathrm{~ms}$ and the subsequent heat release have already been observed in previous time-resolved PIV and OH-PLIF measurements [10, 11]. The additional acetone-PLIF now shows that the vortex has another important effect before the flame roll-up, namely the strong mixing of fuel and air. This provides a homogeneous ignitable mixture, which is required for the subsequent rapid ignition. The vortex-induced mixing thereby contributes substantially to the stabilization of the flame. More generally, the results show that the flame dynamics can only be properly understood when an analysis of mixing mechanisms is included.

\subsection{Mixing, reaction and local extinction at the flame root}

Figure 7 shows a time-series of measurements focusing on the dynamics at the flame root near the unsteady LSP (marked with a dot). Around the LSP, hot burned gas from the IRZ and unburned gas from the nozzle below collide frontally. At $t=0$ $\mathrm{ms}$, the high levels of $\mathrm{OH}$ in this zone indicate that reaction takes place. The unburned gas flowing towards the LSP from below, however, is pure air and thus cannot further sustain reaction. At $t=0.2 \mathrm{~ms}$, the superequilibrium $\mathrm{OH}$ near the LSP has decayed towards equilibrium, indicating that the reaction has been quenched. The quenching of reaction may also be partly caused by the high rates of compressive strain around the LSP of up to $80001 / \mathrm{s}$ plotted to the right. In the subsequent images at $t=0.4 \mathrm{~ms}$ and $0.6 \mathrm{~ms}$, air remains approaching the LSP from below, and no reaction takes place as indicated by the low $\mathrm{OH}$ levels. Starting at $t=0.6 \mathrm{~ms}$ and continuing at $t=1.1 \mathrm{~ms}$, however, a vortex enters from below and transports fuel towards the boundary of burned gas. At $t=1.4 \mathrm{~ms}$, a layer of mixed fuel and air has formed alongside the boundary of burned gas. At $t=1.8 \mathrm{~ms}$, the level of $\mathrm{OH}$ starts rising at the boundary of the burned gas, indicating that reaction takes place again. Subsequently, flame roll-up and heat release take place $(t=2.6 \mathrm{~ms})$ as presented previously in Sect. 5.2. As for $t=0 \mathrm{~ms}$, high $\mathrm{OH}$ levels are present at the flame root at $t=2.6 \mathrm{~ms}$, and subsequently similar processes of quenching, mixing and ignition repeat periodically in conjunction with the PVC motion (not shown).

The dynamics seen in Fig. 7 demonstrates the highly intermittent state of reaction at the flame root. In the $2 \mathrm{D}$ measurement domain, phases of strong heat release $(t=0 \mathrm{~ms} \& t=2.6$ $\mathrm{ms})$ periodically alternate with periods where reaction is locally quenched $(t=0.2-1.4 \mathrm{~ms})$. With respect to the PVC, however, the temporal dynamics seen in Fig. 7 can be interpreted also as a rotation of a $3 \mathrm{D}$ flame structure through the $2 \mathrm{D}$ domain $[8,9,10,27]$. It is therefore assumed that zones of heat release and quenching are simultaneously present in the 3D flame, but appear alternating in the 2D time-series.

\section{Summary and conclusions}

Interactions of flow, fuel-air mixing and reaction in a lean partially-premixed turbulent swirl flame have been investigated 


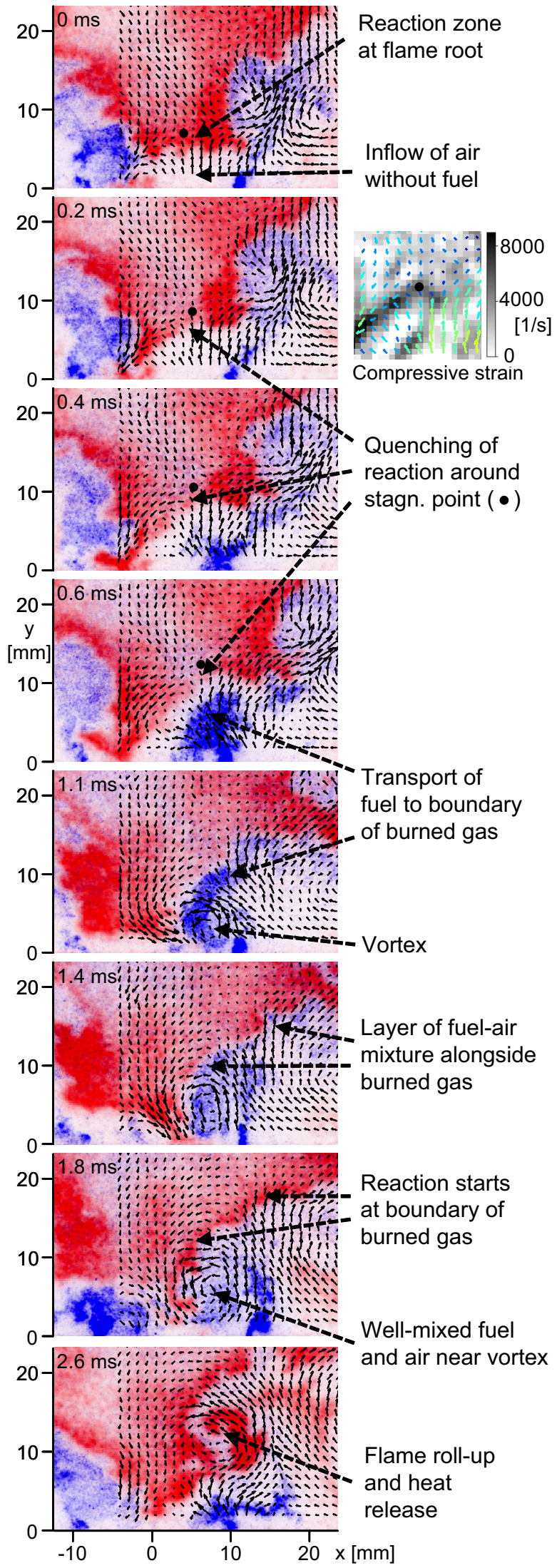

Figure 7: Time-series of PIV, OH-PLIF and acetone-PLIF measurements showing mixing, reaction and local extinction at the flame root. The image at $t=0.2$ ms corresponds to Fig. 5. For clarity reasons, not all time steps are shown. using simultaneous PIV, OH-PLIF and acetone-PLIF with a repetition rate of $10 \mathrm{kHz}$. The flame was operated in a gas turbine model combustor with methane and air at a thermal power of $10 \mathrm{~kW}$ and atmospheric pressure. Transport and mixing of fuel was visualized by adding $9 \%$ by volume acetone to the methane. Comparisons in terms of laminar flame speed [26] and flame shape showed that the acetone-seeded flame is a good representative of the original flame with pure methane.

The major unsteady flow structures are a precessing vortex core (PVC) in the shear layer of the inner recirculation zone and the lower stagnation point (LSP) at the flame root. The measurements show that fuel and air entering the combustion chamber are at first largely unmixed. The PVC, however, periodically passes through the unmixed gas and causes strong mixing. A well-mixed zone of fuel and air forms around the vortex and is then ignited by recirculating burned gas. The PVC then causes a roll-up of the reaction zone, which in turn leads to a strong local heat release. At the flame root, the PVC induces periodic changes in the unburned gas between pure air and well-mixed fuel-air mixtures. It is seen that reaction is locally quenched at the LSP during phases where no fuel is present. Subsequently, well-mixed fuel and air are entrained by the PVC and ignited at the boundary of recirculating burned gas. Generally, the results show that the PVC-induced fuelair mixing contributes significantly to the stabilization of the flame, and that the flame dynamics can only be properly understood when an analysis of mixing mechanisms is included. As mixing depends on Péclet number, which increases with flow velocity, the mixing dynamics for other operating conditions such as those discussed in Refs. [10,11] requires additional studies.

\section{References}

[1] T. F. Fric, J. Propul. Power 9 (1993) 708-713.

[2] W.-P. Shih, J. G. Lee, D. A. Santavicca, Proc. Combust. Inst. 26 (1996) 2771-2778.

[3] T. Terasaki, S. Hayashi, Proc. Combust. Inst. 26 (1996) 2733-2739.

[4] T. Lieuwen, B. T. Zinn, Proc. Combust. Inst. 27 (1998) 1809-1816.

[5] N. Syred, Prog. Energy. Combust. Sci. 32 (2006) 93-161.

[6] M. Freitag, M. Klein, M. Gregor, D. Geyer, C. Schneider, A. Dreizler, J. Janicka, Int. J. Heat Fluid Flow 27 (2006) 636-643.

[7] J. Fröhlich, M. Garcia-Villalba, W. Rodi, Flow Turbul. Combust. 80 (2008) 47-59.

[8] D. Galley, S. Ducruix, F. Lacas, D. Veynante, Combust. Flame 158 (2011) 155-171.

[9] M. Stöhr, R. Sadanandan, W. Meier, Exp. Fluids 51 (2011) 1153-1167.

[10] M. Stöhr, I. Boxx, C. D. Carter, W. Meier, Combust. Flame 159 (2012) 2636-2649.

[11] M. Stöhr, C. Arndt, W. Meier, Proc. Combust. Inst. 34 (2013) 3107-3115.

[12] V. G. McDonell, G. S. Samuelsen, Meas. Sci. Technol. 11 (2000) 870886.

[13] J. Kazenwadel, W. Koban, T. Kunzelmann, C. Schulz, Chem. Phys. Lett. 345 (2001) 259-264.

[14] P. Weigand, W. Meier, X. R. Duan, W. Stricker, M. Aigner, Combust. Flame 144 (2006) 205-224.

[15] W. Meier, X. R. Duan, P. Weigand, Combust. Flame 144 (2006) 225-236.

[16] I. Boxx, M. Stöhr, C. Carter, W. Meier, Combust. Flame 157 (2010) 15101525 .

[17] A. Widenhorn, B. Noll, M. Aigner, Proc. ASME Turbo Expo (2009) GT2009-59038.

[18] M. Stöhr, I. Boxx, C. D. Carter, W. Meier, Proc. Combust. Inst. 33 (2011) 2953-2960. 
[19] Y. Hardalupas, M. Orain, Combust. Flame 139 (2004) 188-207.

[20] R. Sadanandan, M. Stöhr, W. Meier, Appl. Phys. B 90 (2008) 609-618.

[21] A. Lozano, B. Yip, R. K. Hanson, Exp. Fluids 13 (1992) 369-376.

[22] A. K. Prasad, Exp. Fluids 29 (2000) 103-116.

[23] M. C. Thurber, F. Grisch, B. J. Kirby, M. Votsmeier, R. K. Hanson, Appl. Optics 37 (1998) 4963-4978.

[24] B. Yip, M. F. Miller, A. Lozano, R. K. Hanson, Exp. Fluids 17 (1994) 330-336.

[25] C. J. Brownell, L. K. Su, Meas. Sci. Technol. 22 (2011) 085402.

[26] C. T. Chong, S. Hochgreb, Combust. Flame 158 (2011) 490-500.

[27] J. P. Moeck, J.-F. Bourgouin, D. Durox, T. Schuller, S. Candel, Exp. Fluids 54 (2013) 1498. 